\title{
MAMMOGRAPHY LESION DETECTION USING FASTER R-CNN DETECTOR
}

\author{
Reza Reiazi ${ }^{1}$, Reza Paydar ${ }^{2}$, Ali Abbasian Ardakani ${ }^{3}$, Maryam \\ Etedadialiabadi $^{4}$ \\ ${ }^{1,3}$ Department of Medical Physics, School of Medicine, Iran University of \\ Medical Sciences, Tehran, Iran \\ ${ }^{2}$ Department of Diagnostic Radiology, School of Allied science, Iran University \\ of Medical Sciences, Tehran, Iran \\ ${ }^{4}$ Department of Radiology, Milad Hospital, Tehran, Iran
}

\begin{abstract}
Recently availability of large scale mammography databases enable researchers to evaluates advanced tumor detections applying deep convolution networks (DCN) to mammography images which is one of the common used imaging modalities for early breast cancer. With the recent advance of deep learning, the performance of tumor detection has been developed by a great extent, especially using R-CNNs or Region convolution neural networks. This study evaluates the performance of a simple faster R-CNN detector for mammography lesion detection using a MIAS databases.
\end{abstract}

\section{KEYWORDS}

Mammography, Convolution Neural Network, R-CNN, lesion

\section{INTRODUCTION}

Breast cancer is one of the most common causes of death for women in many countries. In general, early detection is very important for cancer treatment and can decrease the mortality rate in a grate extent. One of the common used imaging modalities for early breast cancer detection is mammography in which an abnormality can be categorized as either normal, non-cancerous (benign) or cancerous (malignant).Studies have shown that Mammography can reduce $30 \%$ of mortality in women who were undergoing mammography screening $(1,2)$. Unfortunately the limited sensitivity and specificity of the screening mammography needs to improve diagnostic accuracy of mammography. R-CNN has been coined by Ross Girshick and his group at UC Berkeley as one of the impactful development in techniques convolutional neural networks. The purpose of R-CNNs is to draw bounding boxes over all of the objects in a given images following two step training schedules, first classification and then region detection step. Unfortunately RCNN and its descendent fast R-CNN were computationally expensive, and extremely slow. Recently faster R-CNN has been introduced to overcome the somewhat complex training process that both R-CNN and Fast R-CNN encountered by implementing a classification network using $\mathrm{CNN}$ as the region proposal mechanism for training and prediction steps. This network reduces the overall training pipeline to just check the final convolutional feature map and produce region 
proposals from that. From that stage, the same pipeline as R-CNN is used (ROI pooling, FC, and then classification and regression heads).In this study, we conduct an experiment using faster R$\mathrm{CNN}$ to detect breast abnormalities as normal, benign and malignant.

\section{MATERIAL \& METHODS}

The proposed methods has been comprised in following steps:(1) Data preparation and preprocessing of input images(2) Design and train a faster R-CNN detector,(3) Evaluate Detector Using Test Set. Overall process of this work has been illustrated in figure 1.

\subsection{Data Preparation And Pre-Processing Of InPut Images}

This study used mammography images from a public dataset of the mini Mammogram Image Analysis Society (MIAS) (3). This database includes of 332 mammograms in 1024 x 1024 sizes. It we just used 102 cases which was abnormality (benign or malignant).For the region detector training we have used Matlab Training Image Labeler app to produce required dataset. All cases were labeled in malignant, benign, calcification benign and background (figure 1).

\begin{tabular}{|c|c|c|c|}
\hline $\begin{array}{c}1 \\
\text { imagefilename }\end{array}$ & $\begin{array}{c}2 \\
\text { Benign }\end{array}$ & $\begin{array}{c}3 \\
\text { Malignant }\end{array}$ & $\begin{array}{c}4 \\
\text { Calcification_Benign }\end{array}$ \\
\hline 'G:'|mamography Faster R-CNNMMiasdatabaselmdb063.pgm' & {$[498,526,101,87]$} & [] & [] \\
\hline 'G:|mamography Faster R-CNNMMiasdatabaselmdb069.pgm' & {$[414,579,109,90]$} & [] & [] \\
\hline 'G:|mamography Faster R-CNNMMiasdatabaselmdb072.pgm' & [] & {$[261,512,38,58]$} & [] \\
\hline 'G:'(mamography Faster R-CNNMMiasdatabaselmdb074.pgm' & [] & [] & {$[352,541,40,46]$} \\
\hline 'G:'(mamography Faster R-CNNMMiasdatabaselmdb075.pgm' & [] & {$[445,272,49,74]$} & {$[477,523,50,46 ; 567,656,93,58]$} \\
\hline 'G:'(mamography Faster R-CNNMMiasdatabaselmdb080.pgm' & {$[394,844,71,60]$} & [] & [] \\
\hline 'G:'(mamography Faster R-CNNMMiasdatabaselmdb081.pgm' & {$[352,436,228,218]$} & [] & [] \\
\hline 'G:|mamography Faster R-CNNMMiasdatabaselmdb083.pgm' & {$[481,774,144,101]$} & [] & [] \\
\hline 'G:!mamography Faster R-CNNMMiasdatabaselmdb090.pgm' & [] & {$[490,459,64,53]$} & [] \\
\hline
\end{tabular}

Figure 1.Sample labeling data prepared for train faster R-CNN network

Preprocessing step includes region extraction and contrast enhancement. At the next step, the images contrast was enhanced applying contrast limited adaptive histogram equalization (CLAHE) techniques. This improves the image contrast especially at over internal breast structures. Furthermore we have tried to remove redundant information from training images using texture filters and morphology operators. The effect of preprocessing has been shown on figure 2 . Finally dataset were randomly has been divided into training, testing (respectively $80 \%$ and $20 \%$ of the total dataset). 


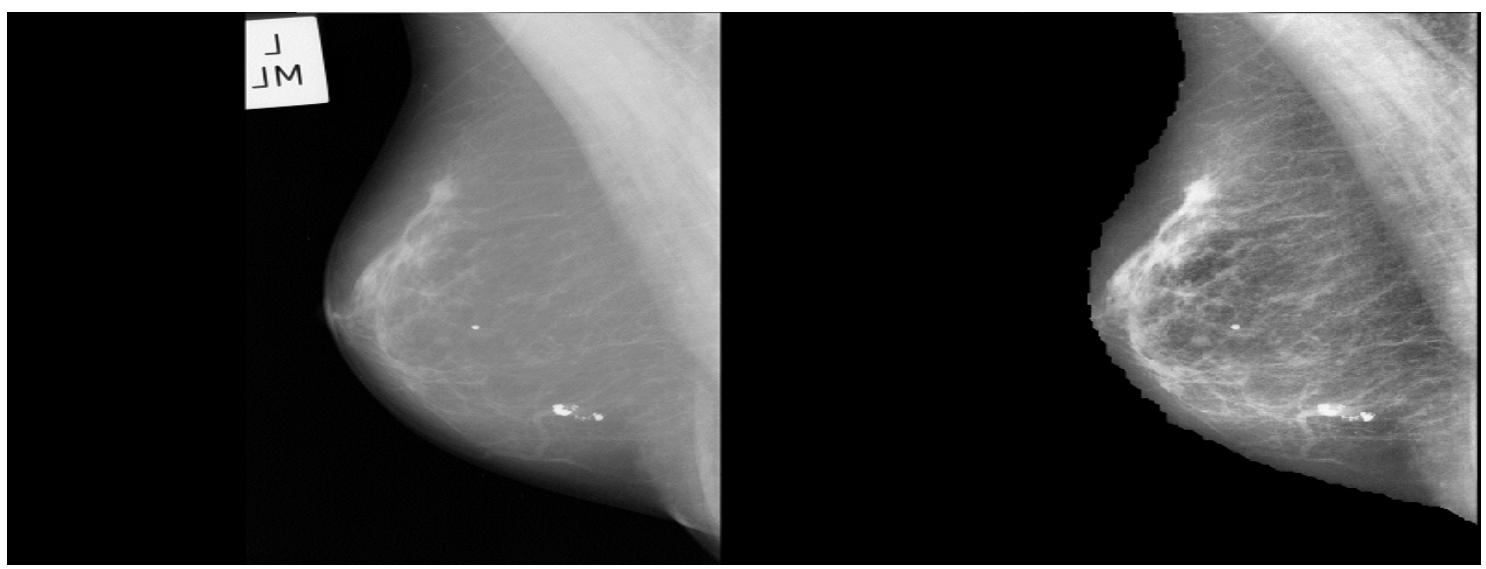

Figure2: the effect of image preprocessing. Contrast of original image (left) has been enhanced using CLAHE algorithm. Also any redundant information has been removed.

\subsection{Design And Train FASTER R-CNN ObJeCt Detector}

The basis of the Faster R-CNN detector is a CNN so called region proposal network. We have design a CNN layer by using Matlab Neural Network Toolbox ${ }^{\mathrm{TM}}$ functionality. The type and size of the training images has been defined by the image Input Layer function. The input size is a trade off between spatial detail that a CNN needs to resolve and processing time which would be very high. For detection tasks, the CNN needs to analyze smaller sections of the image, so the input size must be similar in size to the smallest object in the data set. In this data set an input size of [75 75] has been selected. Building blocks of any CNN is a repeated basic layers of convolutional, ReLU (rectified linear units), and pooling layers follow by fully connected layers, a softmax loss layer and a classification. Repeating a consecutive blocks of convolutional and ReLU with or without pooling layer impose deepness of a CNN. The first convolution layer has a series of 10 filters of size $7 \times 7 \times 3$. One pixel padding has been used to avoid discards of image border information too early in the network. A ReLu layer and max pooling layer (size of 7x7and a stride of 3 pixels) followed the Convolution Layer. The building block of our network has been shown in table 1.

Table1: Building blocks of faster R-CNN network

\begin{tabular}{|c|c|c|c|}
\hline \# & Layer Name & Layer Type & Layer Details \\
\hline 1 & 'imageinput' & Image Input & $75 \times 75 \times 3$ images with 'zerocenter' normalization \\
\hline 2 & 'conv_1' & Convolution & $107 \times 7 \times 3$ convolutions with stride $\left[\begin{array}{ll}1 & 1\end{array}\right]$ and padding $\left[\begin{array}{ll}0 & 0\end{array}\right]$ \\
\hline 3 & 'relu_1' & ReLU & ReLU \\
\hline 4 & 'maxpool_1' & Max Pooling & $7 \times 7$ max pooling with stride $\left[\begin{array}{ll}3 & 3\end{array}\right]$ and padding $\left[\begin{array}{ll}0 & 0\end{array}\right]$ \\
\hline 5 & 'conv_2' & Convolution & $107 \times 7 \times 10$ convolutions with stride $\left[\begin{array}{ll}1 & 1\end{array}\right]$ and padding $\left[\begin{array}{ll}0 & 0\end{array}\right]$ \\
\hline 6 & 'relu_2' & ReLU & ReLU \\
\hline 7 & 'roi pooling layer' & ROI Max Pooling & ROI Max Pooling with grid size [3 3] \\
\hline 8 & 'fc_1' & Fully Connected & 64 fully connected layer \\
\hline 9 & 'relu_3' & ReLU & ReLU \\
\hline 10 & 'fc_2' & Fully Connected & 4 fully connected layer \\
\hline 11 & 'softmax' & Softmax & softmax \\
\hline 12 & 'classoutput' & $\begin{array}{l}\text { Classification } \\
\text { Output }\end{array}$ & crossentropyex with 'Benign', 'Malignant', and 2 other classes \\
\hline
\end{tabular}




\subsection{Evaluate Detector Using Test SeT}

Matlab Computer Vision System Toolbox ${ }^{\mathrm{TM}}$ provides functions for object detector evaluation which calculate average precision. The average precision is a single number that shows the ability of the detector to make correct classifications and the ability of the detector to find all relevant objects (recall).This will be done by running the detector on the test set.
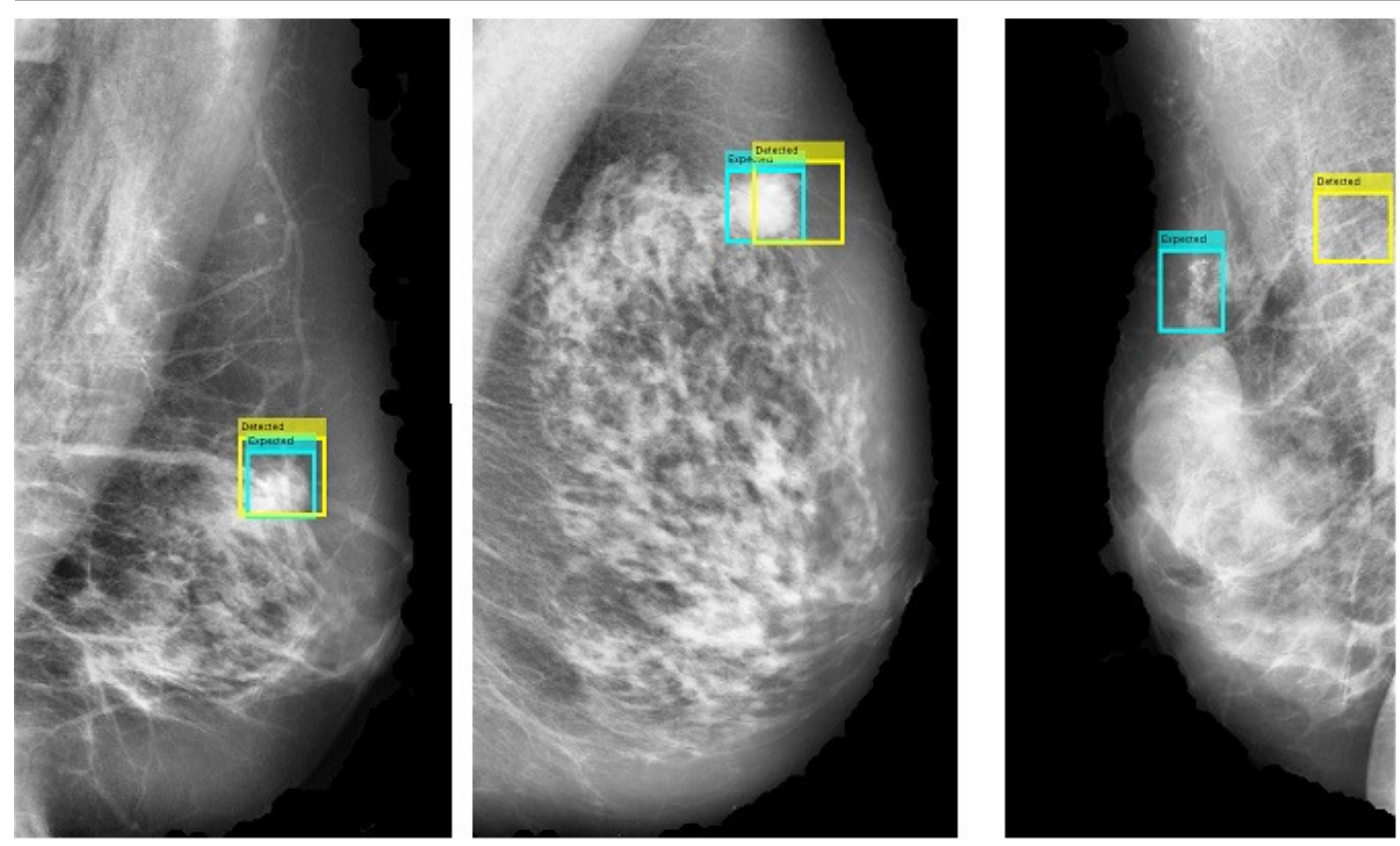

Figure3: Superimposing expected (blue) and detected (yellow) regions on a two malignant case with correct (right), semi correct (middle) and wrong (left) detection

\section{RESULTS \& DISCUSSION}

We have run the trained detector over the test samples to evaluate the precision and recall quantities. Since more than one region with different labels has been detected, the closest box to the original ROI has been selected for evaluation process. If there were more than one closed detected region with different label, we have used that region which has the same label as the test data. Figure 3 has shown the resulting images from tree malignant cases. Ideally, the precision would be 1 at all recall levels. In this study, the average precision is 0.2 . The use of more training data can improve the average precision, at the cost of longer training time.

In some cases despite wrong position detection, there were at least one or more detected malignant regions in 50 percent of malignant (15 from 16 malignant cases) and no detected malignant regions in 94 percent of benign cases (15 from 16 benign cases). This can be due to the similar features in malignant cases and benign cases. The precision/recall (PR) curve highlights how precise a detector is at varying levels of recall. Ideally, the precision would be 1 at all recall levels. The use of additional layers in the network can help improve the average precision. But our results from using deeper network using extra series convolution layer did not improve detection results since the network was not able to detect any region which may impose extra 
layers should be carefully designed and added. We will evaluate this in future applying different designs other that series networks.

\section{CONCLUSION}

Deep convolution neural networks have demonstrated the ability to outperform skilled humans in certain observational tasks $(4,5)$. There are many studies showing reasonable performance of convolution neural networks on mammography lesion detection but none of them uses faster RCNN detectors $(6,7$, and 8). General evaluation studies have shown that an CNN network is able to outperform human performance (9). This study was performed based on Faster R-CNN detector applying a simple series convolution neural network. Trained detector had reasonable detection precision with very small training data (102 images) which can be concluded as

\section{REFERENCES}

[1] Duffy SW, et al, (2002), "The impact of organized mammography service screening on breast carcinoma mortality in seven swedish counties, Cancer, Vol. No. 95(3), pp 458-69.

[2] DB, et al, (2002), "Beyond randomized controlled trials: organized mammographic screening substantially reduces breast carcinoma mortality", Cancer, Vol. 94(2), pp 580

[3] Suckling J, et al, (1994), "The Mammographic Image Analysis Society Digital Mammogram Database Exerpta Medica", International Congress Series, Vol. 1069, pp 375-378.

[4] Giusti R, Gambardella L, Schmidhuber J, (2012), "Neural networks for segmenting neuronal structures in EM stacks", ISBI Segmentation Challenge Competition.

[5] HeK, et al. (2015), "Delving deep into rectifiers: Surpassing human-level performance on imagenet classification", arXiv, 1502.01852,.

[6] Krzysztof J, et al, (2017), "High-Resolution Breast Cancer Screening with Multi-View Deep Convolutional Neural Networks", arXiv,1703.07047v2

[7] Araujo T, et al. (2017), "Classification of breast cancer histology images using Convolution Neural Networks", PLoS One, Vol. 12(6), pp e0177544.

[8] Zhang Q, et al, (2017), "Class Mammogram Classification Based on Descriptive CNN Features". Biomed Res Int", pp 3640901.

[9] Jane H, Anne C, (2017), "Applying Faster R-CNN for Object Detection on Malaria Images", CVPR workshop. pp 808-813. 